\title{
Povidone-Iodine may be the "Silver Bullet" in the Prevention and Control of Covid-19 Infection, Based on New Scientific Data
}

\author{
Ricardo AP Persaud* \\ Consultant ENT Surgeon, Al Zahra Hospital, Sharjah, UAE
}

*Corresponding author: Ricardo AP Persaud, Consultant ENT Surgeon, Al Zahra

Hospital, Sharjah, UAE.

Received Date: July 16, 2020

Published Date: August 07, 2020

\section{Opinion}

The current Covid-19 pandemic is caused by a highly contagious novel coronavirus which originated from Wuhan China in 2019 [1]. Povidone-iodine (P-I) is known to have virucidal activity [2], that is far superior to any of the commonly used antiseptic agents, including chlorhexidine gluconate and benzalkonium choloride [3]. Previous in vitro studies have shown P-I to be effective against coronaviruses responsible for Sudden Acute Respiratory Syndrome (SARS) [4] and Middle East Respiratory Syndrome (MERS) [5]. Very recently, in vitro experiments have demonstrated that P-I is also virucidal against Covid-19 [6]. In my opinion, P-I is the new PPE and its topical usage in nose, oral cavity and pharynx is now one of the best ways to Flatten the Curve of Covid-19, especially in countries like USA, India and Brazil. Furthermore, P-I maybe the "silver bullet" in the prevention and control of Covid-19 infection, based on the recent in vitro studies [6] and personal observations in ENT clinical practice.

Povidone-iodine was discovered in the USA in 1955 during the search for an antimicrobial iodine complex that is less toxic than a tincture of iodine [6]. Povidone is a water-soluble polymer polyvinylpyrrolidone that acts as a vehicle to transport the iodine to its target; it has no anti-microbial properties. The antimicrobial activity of P-I occurs after the free iodine dissociates from the polymer complex [7]. Once in this free form, iodine rapidly penetrates microbes and oxidizes amino and nucleic acids resulting in the disruption of metabolic pathways and destabilization of the structural proteins [7]. The resulting effect is irreversible damage to the pathogen. Side effects with
P-I below $1.5 \%$ are extremely rare and in clinical trials only 2 out of 500 patients showed positive contact sensitivity [8]. The anti-viral properties of P-I may be exploited in many ways in the fight against Covid-19. In general, it can be used as an disinfectant in the nose, pharynx and oral cavity to prevent and control Covid-19 infection as well as transmission. This is because those anatomical regions act as the initial sites of attachment and replication for Covid-19, in both symptomatic patients and asymptomatic carriers $[10,11]$. Active viral replication in the throat has also been confirmed by the presence of viral replicative mRNA intermediates in throat-derived samples [11]. Furthermore, the protease enzyme which is involved in viral replication is also present in the upper oro-digestive tract $[10,11]$. It has recently been found with PCR assay that the nasopharynx has a higher viral load than the oropharynx [12], most probably because the respiratory epithelial lining has the highest expression of ACE2-the main receptors for Covid-19 [13]. Moreover, saliva was found to contain a high viral load in Covid-19 with up to $1.2 \times 10^{8}$ infective particles per ml when samples of patients were analyzed at the time of admission to hospital [14]. For these reasons, most of the PCR routine testing is done using nasal and/or oropharyngeal sampling.

Considering the above, it is logical that any measures to remove or reduce the viral load in nose, pharynx and mouth would not only help to prevent the Covid-19 progression beyond the first phase of contamination, but also reduce community transmission by minimizing aerosol shedding of the infective viral particles whilst sneezing, coughing and talking. Oral rinsing and gargling with P-I 
is well-tolerated and its safety profile has been established with decades of clinical use [15]. Topical nasal application of P-I, below 1.25 percent, is also safe and well-tolerated with no adverse effects on thyroid function, mucosal appearance, ciliary beat frequency/ mucocilliary clearance or olfactory function [16-18].

Based on recent in vitro studies [6], I recommend $10 \mathrm{mls}$ of $0.5 \%$ $(5 \mathrm{mg} / \mathrm{ml})$ P-I oral rinse/gargle for at least 60 seconds twice daily for asymptomatic individuals and four times a day for symptomatic patients. At the moment, there is no commercially available nasal spray containing P-I. However, a $0.5 \%$ of P-I solution can be easily prepared by 1:2 dilution of Betadine (C) mouthwash/gargle which contains $1 \% \mathrm{w} / \mathrm{v}$ P-I. This may then be stored in a dropper container and applied in the nose as nasal drops when necessary. Based on personal experience, I recommend $0.3 \mathrm{ml}$ of $0.5 \%$ of P-I into each nostril twice daily for asymptomatic individuals and four times a day for symptomatic patients. The head down position is ideal when applying nasal drops, for example Mecca position. Healthcare workers should also follow one of the above regimes, depending on their exposure risks to the virus. Those in contact with positive Covid-19 patients or performing procedures in and around the mouth/nose, regardless of Covid-19 status, should use $0.5 \%$ P-I solution four times a day in the nose $(0.3 \mathrm{ml})$ and mouth rinse/gargle ( $10 \mathrm{mls}$ for at least 60 seconds).

In patients undergoing surgical procedures, P-I may help to limit the spread of viral transmission to the surgeon and other theatre staff. The painting of antiseptic P-I solution on the skin before an incision is made has been used for over 60 years and this practice should continue, especially in the current Covid-19 pandemic. All patients having an operation or procedure, with or without general anaesthesia, may undergo the following antiseptic protocols:

1. oral rinsing/gargling with $10 \mathrm{mls}$ of $0.5 \%$ PI solution for at least 60 seconds.

2. $0.3 \mathrm{ml}$ of $0.5 \%$ of PI solution applied in each nostril and left for at least 60 seconds.

If a general anaesthesia is required, the above protocols are recommended prior to intubation, in order to protect the anaesthetist and theatre staff at the outset. Some surgeons, for example ENT, Oral, Maxillofacial, and Dental, may want to reinforce the mucosal antiseptic surgical field by wiping it with a sponge/ gauze soaked in $0.5 \%$ P-I solution after general anaesthesia, assuming prior verbal consent is taken. For otological procedures, 0.5 percent P-I solution can also be used to irrigate the ear, even if a perforation of the tympanic membrane is present.

In summary, the application of virucidal P-I in the nose, pharynx and mouth is safe and may play a significant role in reducing Covid-19 transmission by decreasing viral replication and shedding take place in the upper respiratory tract (the principal reservoir for Covid-19), during the first phase of infection. Blanket usage of mouthwash/gargle $(10 \mathrm{mls}$ of $0.5 \%$ for at least 60 seconds) and nasal drops containing $0.5 \%$ P-I is my own recommendation as an additional adjunct to personal protection equipment (PPE), hand washing, face mask and social distancing to prevent person to person transmission. Public Health Authorities and hospitals worldwide should now consider P-I in their preventative protocols or guidelines, as this may not only be the "silver bullet" in the current Covid-19 pandemic but also be applicable to future flu viral pandemics.

\section{Acknowledgement}

None.

\section{Conflict of Interest}

No conflict of interest.

\section{References}

1. Gobalenya AE, Baker SC, Baric RS et al. (2020) The species severe acute respiratory syndrome-related coronavirus classifying 2019-COVID-19 and naming it SARS-CovV-2. Nature Microbiology 5(4): 536-544.

2. Wutzler P, Sauerbrei A, Klocking R (2002) Virucidal activity and cytotoxicity of the liposomal formulation of povidone-iodine. Antiviral Research 54: 89-97.

3. Kawana R, Kitamura T, Nakagomi O, I Matsumoto, M Arita, et al. (1997) Inactivation of human viruses by povidone iodine in comparison with other antiseptics. Dermatology 195 (Suppl 2): 29-35.

4. Kariwa H, Fujii N, Takashima I (2006) Inactivation of SARS coronavirusby means of povidone-iodine, physical conditions and chemical reagents. Dermatology 212 (Suppl 1): 119-123.

5. Eggers M, Eickmann M, Zorn J (2015) Rapid and effective virucidal activity of povidone-iodine products against middle east respiratory syndrome coronavirus (MERS-CoV) and modified vaccinal virus Ankara (MVA). Infect Dis Ther 4: 491-501.

6. Pelletier J, Tessema B, Westover J (2020) In vitro efficacy of povidoneiodine nasal and oral antiseptic preparations against Severe Acute Respiratory Syndrome-Coronavirus 2 (SARS-CoV-2) 2020.

7. Sneader W (2005) Drug discovery: a history: Wiley, New York, USA, p: 68.

8. Rackur H (1985) New aspects of mechanism of action of povidone iodine. Journal of Hospital Infection 6: 13-23.

9. Lachapelle JM (2005) Allergic contact dermatitis from povidone-iodine: A re-evaluation study. Contact Dermatitis 52(1): 9-10.

10. Gralton A, Tovey ER, Mclaws ML (2013) Respiratory virus RNA is detectable in airborne and droplet particles. J Med Virol 85: 2151-2159.

11. Wolfel R, Corman VM (2020) Virological assessment of hospitalized cases of corovirus disease 2019. Nature 581: 465-469.

12. Zou L, Ruan F, Huang M, Lijun Liang, Huitao Huang, et al. (2020) SARS CoV-2 viral load in upper respiratory specimens of infected patients. $\mathrm{N}$ Engl J Med 382(12): 1177-1179.

13. Sungnak W, Huang N, Becavin C, Marijn Berg, Rachel Queen, et al. (2020) SARS-CoV-2 entry factors are highly expressed in nasal epithelial cells together with innate immune genes. Nat Med 26: 681687.

14. To KKW, Tsang OTY, Chik Yan Yip C, Kwok Hung Chan, Tak Chiu Wu, et al. (2020) Consistent detection of 2019 novel coronavirus in saliva. Clin Infect Dis.

15. Shiraishi T, Nakagawa Y (2002) Evaluation of bactericidal activity of povidone-iodine and commercially available gargle preparations. Dermatology 204 suppl: 37-41. 
16. Panchmatia R, Payandeh J, Al-Salman R, Emily Kakande, Al-Rahim Habib, et al. (2019) The efficacy of diluted topical povidone-iodine rinses in the management of recalcitrant chronic rhinosinusitis: a prospective cohort study. Eur Arch Otorhinolaryngol 276 (12): 3373 3381.

17. Gluck U, Martin U, Bosse B, K Reimer, S Mueller (2007) A clinical study on the tolerability of a liposomal povidone-iodine nasal spray:
Implications for further development. ORL J Otorhinolaryngolaryngol Relat Spec 69(2): 92-99.

18. Reimer K, Wichelhaus TA, Schafer V (2002) Antimicrobial effectiveness of povidone-iodine and consequences for new application areas. Dermatology 204 (Suppl 1): 1:114-120 